\title{
LA ORGANIZACIÓN EN LA UNIVERSIDAD PÚBLICA DE COSTA RICA: UNA PROPUESTA
}

\author{
Ángel Ruiz*
}

Se busca trazar la perspectiva más amplia en que se inscribe la educación superior y en particular la universidad pública para delinear una organización y estructura académicas acordes con semejante visión. Se establecen ejes y principios de organización y además se proponen "áreas" como nivel central de la organización de la vida académica para el mejor decurso de la universidad pública costarricense, entendida esta última como una sola realidad integrada y con propósitos comunes.
A wide international and national perspective of Higher Education is developed in order to sustain a proposal for the internal organization of public Universities in Costa Rica. Organizational axis and principles are established, and the concept of "areas" is proposed as the central structural level in academic life in order to improve public universities' operation. The approach presented here views public universities as being part of one integrated reality in which common objectives are shared.

* Director del Cen ro de Investigaciones Maternáticas y Meta-Matemáticas, Universidad de Costa Rica y académico del Proyecto Apoyo a la Investigación, AIEM-UNA, Escuela de Matemática, Universidad Nacional, Heredia, Costa Rica. 


\section{Introducción}

La época que vivimos supone importantes cambios para las entidades sociales relacionadas con el conocimiento. En particular, y con relieve, esto se aplica a la educación superior, la que se ve obligada a un replanteo de su misión y de sus principales variables de conducta. ¿Por qué este escenario implica una nueva relación de la educación superior con el entorno? ¿Cuáles son las acciones que se plantean para la educación superior? ¿Cómo puede responder, en particular, la universidad pública a esas demandas?

El tema de la organización o estructura universitaria ha sido de los que más se han discutido en las comunidades de las universidades públicas en los últimos años. En los dos últimos congresos universitarios de la Universidad de Costa Rica ocupó un lugar central, y sendas propuestas organizacionales fueron elevadas a la discusión (como en el caso de las propuestas de los académicos Olimpia López y Justo Aguilar, excandidatos a la Rectoría de la UCR). El tema también ha sido central en las discusiones del congreso de la Universidad Nacional (2002-2004) y en el reciente congreso institucional del Instituto Tecnológico de Costa Rica (2002-2003).

En estas discusiones, sin embargo, se ha filtrado en algunos momentos una conducta metodológica inconveniente, en especial en la Universidad de Costa Rica, priorizar la discusión sobre estructura organizativa por encima de aquella sobre la misión y tareas académicas institucionales más amplias. Es decir, en lugar de definir con profundidad y lucidez las grandes perspectivas que el entorno ha colocado a la educación superior en este contexto histórico, y luego pasar a explorar las vías organizativas para responder a esas condiciones y demandas académicas, se ha favorecido una visión administrativa del tema de la organización.

Tal vez este haya sido el énfasis que obedece a la adopción tácita de una premisa, la cual afirma que no hay que modificar la misión y las funciones de la universidad, sino hacer progresar cuantitativamente lo que existe (posiciones sostenidas ya sea por el peso de la tradición, los intereses de gremio, o incluso por la arrogancia institucional) o por el temor al cambio simplemente, lo que genera el predominio de la inercia. No es el propósito nuestro en este artículo abundar en esas razones. Lo que buscamos es ofrecer una propuesta organizacional posible para la academia pública de Costa Rica. Para ello, sin embargo, y en concordancia con lo que expresamos arriba, haremos primeramente un diagnóstico general de los retos y perspectivas que este contexto histórico ofrece a la educación superior. 


\section{El contexto internacional y nacional y las perspectivas de la educación superior}

Podemos de manera resumida mencionar por lo menos tres elementos de nuestro escenario que obligan a cambios en la relación de la educación superior con el entorno social. En primer lugar, se invocan cambios debido al proceso de la mundialización e intensa internacionalización crecientes que condicionan cada dimensión de los quehaceres humanos y, con fuerza, los educativos. Una visión localista o nacionalista está más que nunca absolutamente excluida en la academia. Debe tomarse en cuenta, sin embargo, que es una época de contradicciones. Hay tendencias positivas y negativas (PNUD, 1999). En segundo lugar, debido a una profundización de la crisis en la sostenibilidad ambiental y humana, se plantea un poderoso llamado a la construcción y fortalecimiento de ideas, valores, compromisos globales. La educación superior tanto en las dimensiones formativas como de investigación ocupa aquí naturalmente responsabilidades relevantes que obligan a replantear los curricula universitarios y a reorientar el sentido de muchas investigaciones hacia ese entorno en el cual se juega las condiciones de vida de las generaciones que vienen. (Véase un número especial de la revista Higher Education Policy 2002). Otra razón para esta nueva relación con el entorno son las características de una fase histórica que vertiginosamente se diregen hacia una "edad del conocimiento", en donde este vector se convierte en fundamento de economía (Thurow, 1996), cultura y vida cotidiana. Evidentemente, conocimiento y educación superior poseen una relación íntima privilegiada. Pero, además, experimentamos cambios en la naturaleza del conocimiento (Gibbons, 1984) y su relación con la sociedad, hay una interrelación mayor entre las construcciones cognoscitivas y el entorno social. Las demandas de este último son más directas, e incluso la realización de varias etapas en estos procesos cognoscitivos se debe hacer con el concurso directo de segmentos de la sociedad. Se trata de un momento en que la tecnología juega un papel de pivote, que incluso establece condicionantes vigorosos para la ciencia y la tecnología (como realidad integrada), y por eso ese concurso social adquiere un relieve. La tecnología de nuestro tiempo posee un impacto propio poderoso que permea la mayoría de dimensiones de la vida social y con fuerza extraordinaria la educación y la cultura (Ruiz, 2001 a).

Son muchas las ventajas que este momento histórico "cognoscitivo" ofrece a la educación superior, debido a su cercanía con el conocimiento, tanto en su construcción como en la formación de especialistas que lo manejen. Una de esas ventajas se expresa en la relevancia que ha adquirido la formación superior. Eso explica, parcialmente, la expansión en las matrículas en todo el mundo en este 
sector educativo. Y también la generación de múltiples y diversas opciones para realizar la misión educativa superior. No debe olvidarse, no obstante, que esta misión y el papel efectivo que juegue la institución de educación superior dependerán de muchas variables; no sucede lo mismo en países en desarrollo que en aquellos altamente industrializados. También las tradiciones culturales juegan un papel. Se está, sin embargo, en una situación caracterizada por transformaciones en todas partes para determinar ese papel de cara al nuevo escenario, donde implacablemente existen oportunidades y responsabilidades mayores.

Globalización, internacionalización, urgencia de compromiso con valores éticos, humanistas, ambientales, y papel determinante del conocimiento, nutren un escenario muy general que resalta la acción de la universitas, ya sea que resulte lúcida y edificante o no. Inevitablemente, la educación superior se ve obligada a procesos de adaptación y cambio a las nuevas condiciones en forma radical.

Podemos resumir las principales acciones transversales que este nuevo contexto provocan para la educación superior:

1. La educación debe asumir una política amplia que le permita construir sus quehaceres diversos con un peso creciente de internacionalización y globalización. Eso se aplica a todas sus dimensiones, pero especialmente a los parámetros de comparación de todas las actividades. De igual manera apuntala como forma de organización académica aquella de las redes y afirma los procesos de cooperación de una manera aun más amplia.

2. La formación seguirá siendo una columna central de la vida universitaria, se trata de una tarea clave, y representa una relación con el entorno que nunca deberá debilitarse. Tampoco las acciones de extensión dejan de ser relevantes. El asunto es que las acciones de investigación juegan un papel más significativo e influyente en un escenario que ha hecho del conocimiento su fundamento. Aquí hay que asumir el sentido más amplio de investigación: creación, gestión, transferencia, orientación cognoscitiva y aplicación. Las tareas de formación y extensión se ven influenciadas drásticamente por la investigación. Tanto en los curricula como en el ejercicio directo de las acciones formativas. Su papel es el de un vector director que orienta las perspectivas y condiciona los procesos.

3. De múltiples maneras, la expansión de la formación superior y su diversificación, los requerimientos de mayor conocimiento en los trabajos, empujan hacia una ampliación de los niveles formativos en la educación superior. Es el regreso inevitable del bachiller o licenciado o master a las aulas universitarias. La educación a lo largo de toda la vida (Tünnermann, 1997). Ahora bien, si los niveles de posgrado y las especialidades adicionales 
se vuelven más relevantes esto condiciona drásticamente la organización curricular, el uso de las instituciones, de sus campus, de los medios de enseñanza aprendizaje, de la relación con el entorno, etc. También es inevitable una reforma de la formación en los primeros grados, puesto que la perspectiva de nuevas etapas formativas y sus características obligan a replantear qué formación debe darse desde un principio.

4. Tanto la evolución del conocimiento que rompe las disciplinas radicionales como la necesidad de abordar los problemas del entorno con múltiples enfoques cognoscitivos, empujan hacia una ruptura drástica de las fronteras organizativas académicas que ha tenido la mayoría de universidades. En particular, potencia procesos de des-departamentalización que permitan un flujo de la multi, inter y transdisciplina (Readings, 1996)

5. El desarrollo revolucionario de la ciencia y la tecnología de nuestra época, especialmente las Tecnologías de la Información y la Comunicación (TIC), implica para las instituciones de educación superior su adopción de una manera radical, en todas sus dimensiones. Esto posee profundas implicaciones curriculares, en la organización de todos los quehaceres académicos y en su relación con el entorno social.

6. Un mundo más globalizado aumenta el reclamo por la asunción de criterios internacionales en todos los quehaceres académicos. Por ello mismo plantea ampliar los niveles de calidad de cada uno de ellos. La eEvaluación y acreditación son asuntos clave (Kent et al, 1997). La nueva relación más íntima con el entorno empuja a su vez a una mayor pertinencia social de las salidas profesionales, de las actividades de investigación y extensión y de la misma misión de la institución universitaria. Por supuesto, establecer cuáles deben ser los sentidos renovados de esos conceptos de calidad y pertinencia (UNESCO, 1998) es decisivo: una reconceptualización de estos términos en un mundo globalizado y lleno de demandas del entorno. (No compartimos, sin embargo, el criterio de algunos intelectuales que enfrenta la globalización y la asocian de manera restrictiva al neoliberalismo, además, la condena como un mal que incluso provocaría las necesidades de reformas educativas con base en la eficiencia y la evaluación institucionales; véase Gurdián, 2001).

Estas acciones invocan cambios importantes en varios asuntos que afectan el tipo de evolución académica que ha tenido hasta ahora la universidad pública costarricense en su conjunto, entre ellos: 
- La relación entre los componentes docencia, investigación, acción social, producción (como ejemplo de conceptos y paradigmas del pasado, consúltese Durán et al, 1984).

- El lugar y características de los posgrados; transformación del pregrado; lugar de la investigación (datos sobre la estructura de la investigación en Costa Rica fueron dados por el CONARE-OPES, 1998).

- La revalorización del papel de la especialización y su lugar en los curricula.

- Las metodologías de enseñanza aprendizaje.

- El sentido de los títulos y especialidades.

- El significado de las disciplinas académicas y los cuerpos organizativos que las han integrado.

- La organización de la infraestructura material.

- La relación de la universidad pública con las instituciones, organismos de la sociedad y la comunidad en general, etc.

Se trata de un replanteo amplio y profundo de sus principales variables.

En nuestro país, la Universidad de Costa Rica ocupó todo el espacio de la educación superior hasta la creación en la década de los años setentas del Instituto Tecnológico de Costa Rica, la Universidad Nacional, la Universidad Estatal a Distancia y la Universidad Autónoma de Centro América. El periodo de los años 1970 y 1980 (Ruiz, 1996) fue de expansión de la universidad pública (consecuencias del boom demográfico de la posguerra, ampliación y progreso de la formación primaria y secundaria, incorporación de mujeres en la educación y vida productiva, organización estudiantil, etc.), creando las principales líneas de desarrollo institucional en las universidades públicas (espacios, criterios, énfasis, y comunidades), (Jofré, 1994). A partir de los años noventas se da una expansión de la universidad privada (CONARE-OPES, 1997) que responde a la contracción y sobre todo estancamiento de las finanzas de las universidades públicas, a las consecuencias de un segundo boom demográfico en el país, etc. (Ruiz, 2001 c).

La estructura de la educación superior en el siglo XXI incorpora la universidad pública y la privada de una manera cualitativamente diferente; esta última exhibe una matriculación estudiantil y una generación de egresados mayores que en las públicas. Es decir: la universidad privada tiene más estudiantes y pone en la calle más profesionales. Que haya más estudiantes universitarios y profesionales en la sociedad no es malo, es lo que sucede en todo el planeta, más o menos. Se trata de una perspectiva inscrita en el futuro. La universidad privada ha ayudado a cumplir con esas exigencias sociales por educación superior en un periodo histórico complejo de transición nacional en que se dio contracción 
Globalización,

internacionalización, urgencia de compromiso con valores

éticos, humanistas,

ambientales, y papel

determinante del conocimiento, nutren un escenario muy

general que resalta la acción

de la universitas, ya sea que

resulte lúcida y edificante o no.

Inevitablemente, la educación

superior se ve obligada a

procesos de adaptación y

cambio a las nuevas

condiciones en forma radical. de las finanzas cel Estado (BID, 1996), como parte de los procesos de búsqueda de equilibrio macroeconómico, cambio de modelo de producción y orientación hacia un nuevo estilo de desarrollo nacional (que no se ha decantado todavía de manera definitiva). El país ha apostado a un modelo que potenciará cada vez más el crecimiento de la universidad privada.

$\mathrm{Si}$ bien algunas de las universidades privadas se han comprometido en procesos de progreso de la calidad de sus quehaceres académicos, sin embargo, en su conjunto las 50 instituciones privadas que hay en el país exhiben serias debilidades. Dada la relevancia de la universidad privada

en el presente y futuro nacionales, una de las tareas más importantes que tiene el país es potenciar los mecanismos nacionales que permitan asegurar un control de la calidad en la educación superior, esencialmente privada, y una defensa del estatus del profesional costarricense, un gremio que se ha visto afectado por la presencia de múltiples personas con formaciones universitarias débiles que compiten en el mercado de trabajo. En Costa Rica al igual que en otras partes de América Latina (González et al, 1997) no se asumieron en los años noventas las tradiciones de control de calidad universitaria europeas (restricción en la creación de instituciones) ni las norteamericanas (múltiples sistemas de acreditación), lo que contribuyó notablemente a un deterioro y bastante irracionalidad en el desarrollo de la educación superior privada del país. El Sistema Nacional de Acreditación de la Educación Superior (SINAES), entidad decisiva de la educación superior del país, es reciente en su operación y no posee el peso y apoyo que debería tener. Se puede ver un recuento de la temática de la acreditación en la Universidad de Costa Rica (aunque con un sesgo que sobreenfatiza el papel de la autoevaluación) en el artículo escrito por Aura Barrantes (2001).

Un sistema nacional de evaluación, acreditación y control público obligatorio de las calidades de toda la educación superior se encuentra en el orden del 
día, pero no puecie negarse que se trata de un asunto muy difícil de llevar a la práctica, debido esencialmente a una multitud de intereses sectoriales, y a la acumulación de múltiples errores de parte del Estado costarricense.

En perspectiva, ya al margen de la solución de los problemas de calidad arriba señalados, la universidad privada y la pública de calidad deberán asumir los retos que el escenario plantea para la educación superior. Los espacios académicos y profesionales deberán establecerse con lucidez y pertinencia social y no con base en posiciones dogmáticas en un sentido u otro (una propuesta amplia sobre la reforma universitaria se puede ver en Ruiz, 2001 c). Esto en los siguientes años requerirá reflexión, discusión y acuerdos estratégicos. Las acciones para generar calidad y progreso académico no son iguales en las privadas que en las públicas. Los cuellos de botella son diferentes en cada caso.

En lo que sigue, sin embargo, nuestro propósito no es abordar la estructura de la educación superior como un todo, esas tareas diferenciadas para las privadas y públicas, sino más bien concentramos en las perspectivas de la universidad pública. Se trata de, una vez interpretado el escenario histórico, delinear resumidamente las principales líneas de conducta en nuestra opinión requeridas y sobre ellas la organización posible de esta academia.

En correspondencia con esta situación que describimos arriba, y a manera de síntesis, podemos considerar que las tareas centrales por delante se pueden condensar en dos acciones macro: a) la creación de un marco regulador nacional de la educación superior pública y privada (orientado a asegurar calidad y pertinencia de este estrato educativo), y b) una reforma de la universidad pública que le permita asumir los grandes ejes que el escenario internacional y nacional provoca para la educación superior. Esta última es académica en esencia, y buscaría construir un programa estratégico con base en las consideraciones que hemos hecho, que permita construir el lugar que se requiere para la universidad pública en el país.

En Costa Rica, si bien la evolución de las universidades (y parauniversidades) públicas se ha dado en contextos y con comunidades separadas (Ruiz, 2001 c), es fundamental asumir que el país (por sus dimensiones, recursos y posibilidades culturales y nacionales) puede y debe establecer un plan maestro como una realidad integrada: una estrategia para la educación superior pública como un todo. Que esto pueda tomar tiempo, es apenas un asunto táctico que, por supuesto, dependerá de la conciencia de los dirigentes universitarios, pero también de todas las comunidades académicas. Algunas de las acciones de la universidad pública requerirán el concurso decidido e integrado de todas las universidades públicas, otros el de una de ellas en especial y el soporte de las otras. La organización de las universidades públicas 
deberá poder favorecer el progreso de estos proyectos y líneas de acción. Ala par de la definición y construcción de "nichos" académicos, resultará vital que estas instituciones posean una mayor flexibilidad y libertad internas que puedan nutrir las convergencias interinstitucionales. Si las universidades permanecen hasta ahora como colecciones de decenas de departamentos autárquicos y cerrados, los planes interinstitucionales serán difíciles de llevar hacia delante con éxito.

Ahora bien, las perspectivas que hemos trazado en las páginas anteriores plantean orientaciones específicas en la organización académica, que es lo que queremos subrayar en este artículo.

\section{La organización académica}

¿Qué es lo que le pedimos a una organización para considerarla buena en este contexto? En esencia queremos que esta organización permita y potencie varias dimensiones en la vida universitaria como:

Crear programas y opciones profesionales con facilidad, calidad y pertinencia, prontitud, y adecuados a las demandas sociales (nacionales, regionales, internacionales).

Generar redes de trabajos interinstitucionales, nacionales, internacionales. Construir y desarrollar proyectos de investigación, extensión, producción, con rapidez y eficacia; dar respuestas.

Fortalecer las opciones y escogencias de los estudiantes.

Potenciar el trabajo y responsabilidades de los académicos.

Adaptarse y adelantarse al progreso cognoscitivo.

Incorporar fácilmente las nuevas tecnologías de manera transversal.

Dar respuestas académicas a la transdisciplinariedad de la evolución moderna del conocimiento.

Generar el establecimiento de lazos con el entomo social de manera natural. Integrar procesos de gestión de la calidad de forma permanente. Hacer que los tiempos destinados por los académicos y estudiantes a loas aspectos administrativos sean mínimos y que el mayor tiempo pueda ser destinado a la vida y producción académicas.

Si es esto lo que pedimos de la organización universitaria, cuáles serían las calidades o las palabras clave que se invocan. Pensamos que: flexibilidad, libertad, interacción y temporalidad. Es decir, se trata de potenciar la flexibilidad frente a la rigidez institucional, aumentar los niveles de decisión individuales, los contactos creativos entre los miembros de la academia, y dejar de pensar en agrupaciones 
y organizaciones para siempre, definitivas, e inmutables. Todo esto se inscribe dentro de las demandas de la nueva relación con el entorno.

¿Cuáles son los ejes de acción en la organización o estructura académica que responda a esta visión académica y a las perspectivas que hemos trazado para la educación superior? Consideramos que ocho ejes pueden condensar la orientación organizativa que pensamos.

Ruptura con departamentos estancos y rígidos. Las universidades públicas del país están llenas de escuelas o departamentos soberanos cuyos lazos de intercambio entre sí son en esencia fortuitos o dependen mucho de quienes estén al frente de los mismos. La organización general de la universidad no favorece la interacción. Romper o debilitar sustancialmente el funcionamiento casi "feudal" de los departamentos es una condición esencial para la promoción de la multi, inter y transdisciplina. Solo una visión flexible que supere las debilidades o dimensiones negativas de los "feudos" departamentales puede generar eficacia en la acción académica integrada institucionalmente; solo así se podrá potenciar la labor del académico dotándole de más posibilidades y libertad, y permitir dar respuestas múltiples y eficaces a las demandas del entomo colectivo. Este es un problema de fondo y difícil de resolver en tanto la departamentalización ha sido una premisa de la organización académica nacional e internacional, y existen numerosos intereses individuales y colectivos colocados en contra del cambio.

Eficiencia en la organización de la toma de decisiones. El principio de partida es: simplicidad, lo que se puede traducir en la existencia de procedimientos con el menor número de pasos posibles y pocos y claros niveles jerárquicos (por eejemplo, sin duplicaciones de líneas de decisión). En la organización actual, los académicos y las unidades existentes poseen múltiples "jefes" y varias líneas de jerarquía sobre sus cabezas. Eso es simplemente inconveniente.

Eficiencia y eficacia en los fundamentos administrativos de la acción académica. La eficiencia es un reclamo de una época que potencia la competitividad, y en la administración de las instituciones de educación superior es un requisito. Esto refiere a los servicios, infraestructura, al uso amplio e inteligente de las TIC, la modernización de procesos, y muy importante la selección de personas de calidad comprometidas con la eficiencia.

Menor politización en la vida institucional. Aunque los espacios de vida democrática constituyen una de las contribuciones de la universidad latinoamericana a la vida académica, muchas veces se ha llegado a una saturación en los procesos de escogimiento de autoridades, debilitando no solo la eficiencia institucional sino también su eficacia. Cuantitativamente hay muchos procesos: de director, subdirector, decano, vicedeano, consejos 
institucionales, rector, etc. Pero hay aquí especialmente asuntos cualitativos. Muchas veces este escogimiento de autoridades da pie para el manejo inadecuado de grupos de influencia, mercadeo y clientelismo electorales, y otras debilidades que conspiran contra el buen desarrollo académico. Se trata, entonces, de buscar un equilibrio entre las necesidades de participación democrática con una reducción de espacios de elección.

Menor endogamia en el reclutamiento de personal. En la misma dirección se inscribe el reclamo por romper con la dominante endogamia que caracteriza la selección de profesionales en nuestras universidades. La prioridad es ahora lo interno, sin dar muchas opciones a lo externo. El compadrazgo y la visión de corto alcance se privilegian. Se debilitan entonces las posibilidades de las nuevas ideas, los nuevos puntos de vista, las formaciones distintas, la ruptura con los nefastos favoritismos locales, etc. En un escenario caracterizado por la globalización e internacionalización, la competitividad intensa, la eficacia, para poder responder a las necesidades del país, la sociedad y la comunidad, abrirse a la contratación de académicos procedentes de otras instituciones y latitudes enriquecería significativamente nuestros horizontes y posibilidades; más que eso representa una auténtica necesidad.

Subordinación de lo administrativo a lo académico. Por razones que se hunden en la historia de los últimos 50 años, muchas de nuestras instituciones todavía exhiben un peso muy grande en las dimensiones administrativas en detrimento de las académicas. Esto se refleja en diferentes dimensiones de la vida institucional: prioridades en los trámites, diferencias en las escalas salariales, entre oros. Esto debe invertirse drásticamente.

Predisposición a la creación de redes y vínculos positivos con el entorno. La construcción de redes y de relaciones estrechas con el entorno muchas veces se ven limitadas y obstaculizadas por la organización interna de las universidades. No resulta fácil ni para los académicos ni para la sociedad establecer acciones con prontitud, eficiencia y eficacia. La situación óptima debería ser exactamente al revés: una organización activa y agresiva con el entorno.

Promoción de la participación de la sociedad en la universidad. No solo porque la universidad debe asumir la rendición de cuentas como uno de sus principios (en particular a la sociedad de la que es parte), sino porque no hay manera de establecer una relación edificante con el entorno si esta es unilateral: de la universidad hacia fuera. Es esencial que haya participación de la sociedad en la estructura institucional de una manera más estrecha y desprejuiciada. Esta se debe aplicar incluso en la organización de las decisiones de la universidad. Los universitarios en Costa Rica, con razón o no, han sido muy defensivos en relación con la participación significativa de la sociedad en sus acciones y decisiones. 
En respuesta a estos ejes de organización de la vida académica, desde un punto de vista teórico, siempre es posible pensar en varias opciones de organización. Por ejemplo, en un modelo organizacional matricial en el cual la relación entre unidad o programa académico sea casi directa con el gobierno central de la institución, un debilitamiento de instancias intermedias. Aquí los académicos pertenecen a la universidad y esta en general organiza su quehacer con programas que aglutinan académicos de varias escuelas, los programas no son permanentes. Puede concebirse la matriz generada en sus filas por programas (interescuelas o institucionales) y en sus columnas por las escuelas existentes. Esto tendría como ventaja una mayor interactividad de la universidad como un todo, y menos jerarquías o niveles estructurales. En el caso de universidades pequeñas esto sería posible sin mucho problema. No obstante, hay desventajas. El esquema matricial puede asumir o no la departamentalización; en el primer caso, no se gana mucho en cuanto a la libertad para los académicos, y en el segundo quedarían programas sin más, se debilitan las disciplinas es difícil de manejar (en particular porque se genera una dispersión muy amplia en la organización). Se requeriría de múltiples recursos materiales y humanos para que este modelo pueda tener éxito. Pero, además, en Costa Rica, en cualquiera de las universidades existentes, intentar pasar de lo que existe a un modelo matricial resultaría muy complejo. Las universidades son bastante grandes y la disposición hacia el cambio aunque no es idéntica en todas las instituciones, no es muy amplia. Tal vez el Instituto Tecnológico de Costa Rica podría obtener mejor provecho de un modelo semejante, pero para instituciones como la Universidad Nacional y la Universidad de Costa Rica sería muy difícil e incluso, probablemente, contraproducente. En esta propuesta nos referiremos a las universidades con modalidad presencial, se excluirá a la Universidad Estatal a Distancia por que posee otra perspectiva organizativa debido a la modalidad de su enseñanza.

¿Qué otras opciones podrían darse? ¿Cómo integrar la movilidad, flexibilidad, libertad y "temporalidad" de los agrupamientos, programas, y opciones académicas que se sugieren aquí sin que se genere la atomización institucional, se favorezca la cohesión de los quehaceres de la universidad, y sin que se rompa abruptamente el tejido social involucrado? ¿Cómo equilibrar estos influjos globales de cambio y permanencia que afectan la universidad en nuestro contexto actual?

Amén de otras posibles orientaciones teóricas, pensamos que la línea de conducta más pertinente (correcta y factible) sería la de fortalecer el nivel de organización intermedio entre la escuela o departamento actuales y el gobierno central (ejercido por la rectoría y sus vicerrectores) o los consejos institucionales (consejos universitarios). La propuesta apunta a la construcción de una forma 
de organización que podemos llamar con el téminino de área. En términos sencillos y de manera abstracta, es en esencia un conjunto de unidades académicas o programas académicos, que se establecen, ya sea por razones de naturaleza cognoscitiva, histórica, académica, funcional, etc. También podemos considerar un área como un conjunto de académicos de diferentes formaciones que realizan su labor esencialmente con programas y pueden agruparse en unidades académicas como las escuelas o departamentos dentro de esa área. Las áreas se pueden llamar facultades o como se quiera, dependiendo de variables históricas o conveniencias sociológicas. En lo que sigue vamos a usar solamente el término área para esta forma de organización.

El área es el marco organizativo en el cual se realizarían, tendrían interacción y se nutrirían los quehaceres de los diferentes académicos asociados al mismo. Cada profesor de la universidad estaría asociado a un área dentro de la cual habría varias unidades académicas o programas de docencia, investigación, acción social, producción, venta de servicios, etc. Es posible pensar, sin embargo, que un profesor participe en uno o varios programas o unidades académicas en diferentes momentos o simultáneamente. Las unidades académicas son agrupaciones de académicos (más permanentes) que tienen a su cargo proyectos de investigación o acción social o producción, o programas de formación. No obstante, en este modelo los proyectos o programas académicos no necesariamente están dentro de una unidad académica. Por otro lado, puede haber académicos que no estén en una unidad académica.

En este modelo, las áreas asumen la coordinación, soporte y gestión específica para los programas y unidades académicas que contiene.

La universidad descentraliza el presupuesto y le da a las áreas un monto apropiado del que pueden disponer plenamente de acuerdo a sus planes de desarrollo estratégico. El área es la que toma las decisiones en torno a la distribución del presupuesto de cada programa y unidad académica en su seno; asume la evaluación y fiscalización de programas y unidades (rendición de cuentas). El nivel superior de autoridad institucional se relaciona directamente con el área, no con los programas o unidades de la misma, y estos últimos directamente con el área. La línea jerárquica de las instancias de organización y mando es: nivel superior, área, programa o unidad.

El área toma las decisiones básicas en torno a la creación de nuevas unidades académicas y los programas académicos (que ofrecen carreras profesionales u organizan la investigación o la extensión). Todos los proyectos y programas formativos de las unidades o académicos en el área, pertenecen a esta.

Las áreas que se proponen aquí serían algo así como las facultades y las unidades académicas como las escuelas, centros e institutos de investigación, 
etc., existentes er: algunas de nuestras universidades públicas, pero sin la departamentalización, e incorporando funciones que están en otros niveles de las estructuras existentes. Debe quedar claro, se propone una des-departamentalización parcial de los que existe, cuyas fronteras las daría precisamente un área, que se ha visto además potenciada.

Varias de las tareas de las áreas se llevan a cabo hoy en entidades centralizadas institucionalmente y otras en escuelas o departamentos. Asume tareas de unos y otros, y nuevas.

Podemos dar unas cuantas pinceladas adicionales para expresar nuestra visión. Vamos a usar el término de decano o decana para indicar la dirección académica de las áreas. Los decanos de áreas se vuelven las autoridades centrales en la vida académica ordinaria de la institución. Los directores de las actuales escuelas o departamentos pasan a ocupar una función de coordinación académica, están subordinados a la dirección de las áreas o facultades, y en esencia son designados o elegidos por los decanos o por un cuerpo directivo de área (con procedimientos académicos apropiados). Estos directores pueden no ser de la institución ni de otras instituciones nacionales. Las plazas de académicos, otro ejemplo, pertenecerían a las áreas o facultades, etc., y las decisiones definitivas sobre la organización de la actividad de cada académico quedarían en manos del decano o decana (con procedimientos que involucran las unidades o programas). Igual se aplica a centros, institutos, etc. La docencia, investigación, acción social y producción de cada académico sería evaluada por su área de manera integrada.

Cada área debería tener un conjunto de plazas de uso temporal para académicos visitantes (reales, no como sucede en la UCR que se usa esa modalidad como simple trampolín de ingreso en Régimen Académico), que pueden darse a profesionales de otras instituciones o empresas nacionales o extranjeras, y las áreas generarían sistemas de contratación que permitan incorporar en propiedad intelectuales extranjeros de calidades reconocidas.

Habría otros asuntos a considerar y detallar en la nueva organización: ¿Cómo se elige un decano o decana? ¿Qué persona con la existencia de cuerpos de dirección del área con la participación de los directores, coordinadores de programas, etc.? ¿Cuáles criterios se darán para la construcción de unidades o programas académicos y su permanencia? ¿Cuáles serán los nuevos mecanismos para la contratación y permanencia del personal? ¿Cómo acomodar los posgrados y la investigación en cada caso? ¿Cómo hacer la rendición y la revisión de cuentas individual dentro de las áreas? Y muchos otros. Pero con lo expresado hasta ahora ya hemos delineado la idea básica de la propuesta organizativa, y con base en ella no sería difícil encontrar respuestas coherentes para cada una de las preguntas pregunta de arriba. 
Existen dos principios subyacentes en esta propuesta: por un lado, la idea que la organización más básica debe ser temporal (no indefinida) de los académicos, puesto que entre otras cosas se plantea con mayor intensidad y regularidad la revisión de pertinencias de los quehaceres académicos debido a las demandas de un entomo tan cambiante, la rendición de cuentas, la evaluación, la comparación para lograr calidad, etc. La desdepartamentalización va en ese sentido. Pero, para evitar una dispersión o atomización institucionales, se crean instancias intermedias, que sin embargo deben ser fuertes. Esto conduce a un segundo principio involucrado: la descentralización de la institución, que obedece a políticas necesarias para el desarrollo institucional dada la evolución histórica de la universidad pública en Costa Rica. Son dos líneas de acción que tienen que ir juntas: una descentralización efectiva (presupuestaria, académica, etc.) de toda la universidad y una concentración de los quehaceres académicos en las áreas o facultades con base en la desdepartamentalización de la vida universitaria. Si se pretendiera descentralizar sin desdepartamentalizar se cometería un error, porque en muchas ocasiones las entidades centralizadas han sido buenos contrapesos a las acciones inapropiadas de los directores de escuelas o departamentos o de las posiciones de poder de las mayorías (asunto que a veces los "descentralizadores" a ultranza no han comprendido). La descentralización y departamentalización juntas representarán siempre un mayor poder para los feudos, y dada las formas de decisión electorales que existen, iría en detrimento de las minorías y de los académicos individuales. Otro error sería descentralizar las finanzas al margen de cambios académicos más de fondo. Algunas de estas orientaciones, en nuestra opinión son erróneas, sin embargo, han sido propuestas en los recientes congresos universitarios de Costa Rica.

La universidad, en nuestra visión, se organizaría entonces esencialmente con base en estas áreas o facultades y desconcentrarían y descentralizarían (en diferentes dimensiones) su actividad global. El área o facultad se convierte en la unidad o nivel académico fundamental de la institución, la cual puede verse como la integración de varias comunidades académicas.

La universidad en este modelo no es, sin embargo, una simple federación de áreas: se dan acciones y procesos que trascienden las áreas. Se trata de acciones estratégicas que convienen a todas las áreas y que por separado no se podrían realizar apropiadamente, así como mecanismos que establezcan parámetros y criterios de calidad uniformes, acciones de fiscalización administrativa, espacio para la realización de procesos de apelación a decisiones tomadas dentro del área, acciones de evaluación institucional, nacional, internacional, acciones de relación global con el Estado y la sociedad civil (que pueden incluir entre otras cosas: negociaciones presupuestarias, rendición de cuentas, coordinaciones con 
otras instituciones, coordinación de la participación en los planes nacionales de desarrollo, etc.). Por otro lado, la integración de las áreas es relevante para potenciar la visión humanista que debe ser consubstancial a la universitas, y que consideramos es una premisa para estas instituciones en el país

Todo esto significa,que temas de interés global pueden y deben ser atendidos por la universidad como un todo. Es posible que, incluso, asuntos como los posgrados y la investigación en las instituciones que poseen menos fortalezas en ellos, deban ser apoyadas institucionalmente y no dejadas en condiciones adversas cuando, por ejemplo, un área no posea la madurez para asumir ese tipo de tareas. El gobierno central de la universidad deberá tener recursos materiales y humanos para favorecer acciones especiales de interés institucional (por ejemplo, plazas para proyectos transdisciplinarios, posgrados especiales, proyectos de acción social relevantes, etc.). Incluso es posible pensar en entidades institucionales con vida independiente, aunque con compromisos con las áreas.

Las posibilidades de acciones inter-áreas, o simplemente transdisciplinarias, deben encontrar apoyos institucionales especiales (bolsas de investigación, extensión, cultura, arte, etc.). Sería fácil favorecer la transdisciplina con una organización más simple que lo que ahora suponen decenas y decenas de escuelas separadas y estancadas. Insistimos en esto: hay que ofrecer las oportunidades a los académicos para realizar apelaciones a decisiones tomadas en el área. Dentro de la perspectiva que sugerimos aquí se requeriría replantear el papel de los organismos centralizados técnicos para poder ofrecer apoyos administrativos y académicos comunes para todas las áreas. Por ejemplo, procesos de información, registro, admisión, etc.

De alguna manera, la descentralización y "empoderamiento" de las áreas debe complementarse con un fortalecimiento de acciones institucionales centralizadas estratégicas, en particular, la gestión y la evaluación.

De igual forma, aunque no vamos a entrar en detalles sobre esto, es necesario señalar que una organización de la universidad con esta mentalidad supondría cambios profundos en todos los niveles políticos. El nivel superior de autoridades universitarias, por ejemplo, debería redefinirse. La inevitable coordinación de áreas invoca, de manera apenas natural, una instancia colegiada de los decanos de área que debería realizarse en conjunción estrecha y armónica con el gobierno central (es decir, con la participación oficial de miembros de la administración central), para orientar y desarrollar los quehaceres académicos de la institución como un todo. En otras palabras, una instancia integrada de puente o engranaje entre la vida ordinaria académica realizada en las áreas y la administración, gestión y política institucionales como un todo. Solo los decanos federalizarían la institución, y se impedirían las coordinaciones esenciales con la 
administración central. En una estructura como esta varias de ias tareas que hoy asumen la administración central o los consejos "institucionales" o "universitarios" en las universidades públicas costarricenses (tareas "ejecutivas" y "legislativas" podrían salir de la Rectoría y de los consejos universitarios de hoy respectivamente) se podrían colocar dentro de esa nueva instancia, y eso mismo, sin duda, plantearía un nuevo sentido y función para la Rectoría y esos consejos, hacia tareas globales, estratégicas y de relación con el entorno social nacional e internacional. El modelo que involucra a tres entes: el Consejo Universitario, la Rectoría y el Consejo de Decanos, que, por ejemplo, existe en la UNA y que se ha propuesto el año 2003 en el congreso universitario de la UCR (aunque con diferencias entre ambas instituciones), cambiaría drásticamente si la base de la organización son las áreas en el sentido que hemos propuesto en estas páginas, y podría mejorar algunas fallas de la estructura existente en estos niveles (véase para el diagnóstico de la UNA, Miranda, 2002).

En la UCR, en los últimos años, se han dado opiniones en una dirección similar, aunque no con todos los elementos que sostenemos en nuestra propuesta afirmando la necesidad de potenciar los niveles intermedios de organización y autoridad, es decir, las facultades existentes. Esto lo recoge Olimpia López (2004) que resume posiciones de los decanos de esa institución:

Los decanatos deben fortalecerse, pueden asumir asuntos de las vicerrectorías y si se les da poder y autoridad, lograr la interdisciplinariedad en las facultades. Los decanos deben unir las escuelas, coordinar posgrados y recursos, formular lineamientos y políticas académicas. Deben unir y acercar las disciplinas porque eso es lo que se requiere.

A lo anterior se añade lo siguiente:

Además, el proceso decisorio está estratificado y centralizado en el rector y los vicerrectores, se responde más a problemas y demandas de corto plazo que a una verdadera planificación. Por eso la Universidad no reacciona rápido a las demandas del entorno, tampoco a las internas. Hay que achatar la estructura, disminuir los niveles jerárquicos para reducir la burocracia; decanos o vicerrectores, no ambos. Menos jefes y más trabajo. Los decanos pueden ejecutar junto con el rector, puesto que entre ambos hay un juego de relaciones más directo. (López, 2004). 
Alguien pociría pensar, para ir completando este ensayo, que una reforma en esta dirección sería simplemente pasar de unos "feudos" a otros más grandes. En efecto, no se propone aquí una desdepartamentalización absoluta. Sin embargo, ya simplemente el cambio cuantitativo que se sugiere generaría un auténtico salto cualitativo. Se trata de un funcionamiento que pondría en relación entre sí un número más grande de académicos (ahora encerrados entre los barrotes de las escuelas), potenciaría intercambios y procesos transdisciplinarios dentro de las áreas, aumentaría los espacios y posibilidades para el movimiento de los académicos, etc. Por otra parte, al reducir el conjunto de "feudos" y "señores feudales", haría más simple la relación entre el nivel superior de la política institucional y los quehaceres académicos. Las líneas jerárquicas se simplificarían (un director o coordinador de escuela debe responder solo al decano o decana). Pero hay más que reducción del número de feudos; por ejemplo, al fortalecerse los procesos de fiscalización, evaluación y gestión centralizados se puede contrapesar el lugar de las autoridades del área en beneficio del académico individual y la institución en su conjunto. Incluso, los procesos administrativos se podrían realizar de manera más eficiente por área.

Este modelo, en síntesis, genera varias líneas de conducta en relación con lo que existe:

- Descentraliza la universidad, traslada competencias del gobierno central a las áreas.

- Fortalece el nivel intermedio de organización institucional.

- Restringe las competencias de las escuelas o departamentos.

- Fortalece las competencias de las autoridades superiores en tareas estratégicas como la gestión, la evaluación, la fiscalización, etc.

Hemos dejado para el final un asunto clave: ¿cuáles pueden ser los criterios para definir las áreas en cada universidad? En esto me parece que lo mejor es tener una amplitud metodológica: unas pueden estar definidas por un criterio (por ejemplo cognoscitivo), otras por el contexto (conveniencias para potenciar un tema clave), otras por razones de comunidad universitaria o trayectoria histórica (el peso de lo que existe), etc. El criterio cognoscitivo debe tomarse en cuenta con mayor relieve; muchas de las facultades que hoy existen en nuestras universidades obedecen a esos parámetros, sin embargo, los tiempos, el conocimiento y su estructura interna han cambiado mucho; esto plantea incursionar en nuevas posibilidades de agrupación cognoscitiva distintas a las tradicionales disciplinas que hoy subsisten. Pero, la discusión no se puede hacer en el vacío, porque se trata de entidades sociales vivientes. Las áreas en cada institución deberían redefinirse o 
restablecerse con base en criterios diferentes. En instituciones más pequeñas o más dispuestas al cambio puede pensarse en cambios más drásticos para consuruir áreas muy novedosas. Es posible, no obstante, que instituciones más rígidas y tradicionales como la Universidad de Costa Rica, lo que le convenga sea no modificar tanto la estructura actual sino más bien las funciones de cada componente con base en algunos de los principios que hemos sugerido en estas páginas. En la Universidad de Costa Rica y la Universidad Nacional el sentido de las áreas lo podrían asumir las facultades existentes, con algunas transformaciones adicionales: traslado de algunas escuelas y departamentos hacia otras facultades, creación de nuevas áreas, agrupación de algunas facultades, etc. Es decir, sin cambiar drásticamente las facultades existentes, se las podría dotar de una naturaleza distinta en su funcionamiento organizativo.

En este marco organizativo que hemos sugerido se puede pensar de cara al futuro en mayores y más amplias interacciones, intercambios, programas académicos que trasciendan los límites de una sola institución.

El momento histórico puede ser apropiado para realizar algunas reformas en cada institución con base en el lugar de cada universidad dentro de la estrategia de la entidad pública como un todo. Esto es relevante: es pensar en la perspectiva de que cada universidad debe ocupar un lugar propio dentro de la estrategia integrada de la universidad pública del país. Para eso se requiere, como paso previo, definir con precisión las fortalezas, vocación y oportunidades estratégicas para cada universidad. Apela a la visión y la misión de cada institución. La Universidad de Costa Rica es la institución con una oferta académica más universal; la Universidad Nacional y el Instituto Tecnológico de Costa Rica han desarrollado ciertos énfasis: ciencias sociales, letras y educación en el primer caso, ingenierías en el segundo. Sin embargo, hay trabajos muy relevantes y de gran calidad que no se pueden colocar mecánicamente en esos énfasis: sismología y veterinaria en la Universidad Nacional, por ejemplo, administración de empresas en el Instituto Tecnológico de Costa Rica. Esto invoca redefiniciones más amplias, no excluyentes, que deben tomar en cuenta la trayectoria y la visión propias desarrolladas hasta ahora por cada institución.

\section{Conclusión}

El escenario que vivimos se ha modificado drásticamente en los últimos 25 años, tanto en dimensiones políticas y económicas como culturales y sociales relevantes. "Globalización", "sostenibilidad", "edad del conocimiento" son términos que se refieren a transformaciones significativas de las variables que rigen nuestro momento histórico. En esta nueva realidad la educación superior 
posee un papel muy relevante (aunque varía de acuerdo a la región del mundo que se trate) por su intensa relación con el conocimiento, y por la existencia natural de muchas aspiraciones y redes internacionales asociadas que hoy se ven potenciadas drásticamente. En particular, sus perspectivas en la educación superior plantean cambios en sus acciones, en su misión y en sus tareas académicas. Es un fenómeno intemacional que se da también en Costa Rica.

Una nueva relación con el entomo se plantea para la educación superior, que enfatiza la investigación, la transdiciplina, tecnologías de la información y comunicación, globalización e internacionalización vigorosas y dentro de una sociedad que exige educación permanente en toda la vida. El cambio vertiginoso, la intensidad de las transformaciones cognoscitivas, culturales y sociales que vivimos, obliga a una educación superior más amplia con una capacidad de respuesta más rápida, mucha flexibilidad en todos sus quehaceres, generación de una gran diversidad de salidas profesionales, con una interrelación decidida entre los miembros de sus comunidades académicas.

La búsqueda de calidad en la educación superior exige mecanismos de regulación nacionales, muy en especial en relación con la universidad privada, en la que se han tenido serios problemas, que han deteriorado el estatus profesional y que han presionado negativamente los niveles de excelencia académica del país.

En Costa Rica, la universidad pública requiere asumir estos cambios académicos de fondo en sus perspectivas tanto de visión, misión como tareas institucionales, y responder en todas sus dimensiones a los retos de ese futuro. A la vez, la universidad pública debe dotarse de la organización que corresponda a esas condiciones. Para ello deberá tomar en cuenta lo que existe, trayectorias y condiciones materiales y humanas, y abordar cambios de su estructura dentro de lo que debe verse como una sustancial reforma universitaria.

En primer lugar, el país debe trabajar en una mayor integración de las universidades públicas, para potenciar el uso de sus recursos humanos y materiales, y poder dar respuestas integrales y efectivas a las necesidades del país.

En segundo lugar, en cada universidad, proponemos dos procesos en las perspectivas de la organización, por un lado una descentralización de la universidad (en las presenciales), trasladando tareas que durante varias décadas han estado en manos de entidades centrales de esas instituciones. Por el otro lado, concentrando tareas en áreas con base en un proceso de ruptura de la departamentalización, que ha sido hasta nuestros días columna vertebral de la organización de las universidades públicas del país. Se trata de una desdepartamentalización moderada que reduce las debilidades académicas que ha provocado un modelo basado en la existencia de departamentos estancos, modelo que fue valioso y positivo en una etapa de la vida universitaria del país. 
A la vez, se sugiere potenciar tareas estratégicas de manera centralizada como: la gestión, la evaluación, etc.

Estos cambios organizativos solo tienen sentido denro de una perspectiva de reforma universitaria que implicaría entre otras cosas reformas curriculares profundas, nuevas premisas en la contratación y permanencias de académicos, reordenamiento de las variables de docencia, investigación y extensión, apertura real a la sociedad, una cultura de rendición de cuentas, internacionalización drástica de los parámetros universitarios, y un compromiso profundo con la calidad y la pertinencia social de los quehaceres universitarios.

Las posibilidades de una reforma de esta naturaleza dependería de muchos protagonistas: el Estado, la sociedad civil, y los universitarios. Por eso, no es algo fácil de llevar a cabo, aun si se obtuviera consenso en las comunidades universitarias en torno a su necesidad. Sin embargo, hay una circunstancia actual importante que puede favorecer el cambio: la transición generacional que atraviesan todas las universidades públicas, debido a la jubilación masiva de muchos de sus cuadros académicos y administrativos. En esa dirección la inercia que podría existir en quienes fueron formados en ciertas reglas del juego universitario, se vería debilitada por camadas de nuevos académicos y gestores administrativos con la mirada puesta en el futuro, dentro de una época que potencia el cambio cotidiano como nunca en la historia de nuestra especie.

Como nación, finalmente hay que comprender el valor de la inversión en educación superior y apuntalar sus procesos de reforma expresaría una conciencia positiva sobre las grandes variables de nuestra época, y sobre las posibilidades para un país como Costa Rica, que posee importantes condiciones pàra definir su derrotero futuro con base en el uso y construcción inteligentes del conocimiento y la educación.

\section{Referencias}

Banco Interamericano de Desarrollo, BID (1996). A la búsqueda del siglo XXI: nuevos caminos de desarrollo en Costa Rica. San José, Costa Rica.

Barrantes, A. (2001). Autoevaluación y acreditación de las carreras de la Universidad de Costa Rica, Revista de Ciencias Sociales, Universidad de Costa Rica, números 92-93, 2001 (II y III), pp. 77-91.

CONARE-OPES (1997). Diplomas otorgados por las instituciones de educación superior universitaria de Costa Rica, 1990-1996. San José, Costa Rica.

CONARE-OPES (1998). Estadísticas básicas sobre la investigación en las instituciones de educación superior universitaria estatal, 1996. San José, Costa Rica. 
Durán, F. Del Vecchio, J. Macaya, G. y Fonseca, O. (1987). Paradigma de la Universidad de Costa Rica. Ciudad Universitaria Rodrigo Facio, Costa Rica: Publicaciones Universidad de Costa Rica.

Gibbons, M. et al (1984). The new production of knowledge. London: Sage.

González F., L. Ayarza, Hernán (1997). Calidad, Evaluación institucional y acreditación en la educación superior en la región latinoamericana y del Caribe, en UNESCO-CRESALC.

Higher Education Policy (The Quarterly Journal of the International Association of Universities) (2002). Vol. 15, no. 2, June 2002, Tema central: Sostenibilidad y Educación Superior. Los editores invitados fueron: Peter Blaze Corcoran, Wynn Calder y Richard M. Clugston.

Gurdián, A. (2001). Dimensión política de la evaluación y la acreditación. Revista de Ciencias Sociales, Universidad de Costa Rica, números 92-93, 2001 (II y III), pp. 11-22.

Jofré V., A. (1994). La Universidad en América Latina: desafíos y estrategias para las próximas décadas. San José, Costa Rica: Editorial Tecnológica de Costa Rica.

Kent S., R. y De Vries, W. (1997). Evaluación y acreditación de la educación superior latinoamericana: razones, logros, desafíos y propuestas, en UNESCO-CRESALC.

López, O. (2004). La Universidad del Siglo XXI. San José, Costa Rica: Editorial de la Universidad de Costa Rica.

Miranda, R. (2002). Prospectiva de la estructura organizativa en la Universidad Nacional. Heredia, Costa Rica: Programa Gestión de la Calidad Institucional, UNA.

Programa de las Naciones Unidas para el Desarrollo, PNUD (1999). Informe sobre desarrollo humano 1999. Madrid, España: Mundi-Prensa Libros, S. A.

Readings, B. (1996). The University in Ruins. Cambridge, Massachusetts, and London. England: Harvard University Press.

Ruiz, A. (1996). Universidad y sociedad en América Latina. San José, Costa Rica: FLACSO-UNA.

Ruiz, A. (2001 a). El destino de Costa Rica y la educación superior. San José, Costa Rica: EUCR-CONARE. http://cimm.ucr.ac.cr 
Ruiz, A. (2001 b). El siglo XXI y el papel de la universidad. San José, Costa Rica: EUCR-CONARE. http://cimm.ucr.ac.cr

Ruiz, A. (2001 c). La educación superior en Costa Rica. San José, Costa Rica: EUCR- CONARE. http://cimm.ucr.ac.cr

Thurow, L. (1996). El futuro del capitalismo. Argentina: Javier Vergara editor.

Tünnermann, C. (1997). La educación superior frente al cambio. EDUCA/ CSUCA. San José, Costa Rica.

UNESCO (Organización de las Naciones Unidas para la Educación, la Ciencia y la cultura) (1998). "Hacia un Programa 21 de la educación superior", documento de trabajo para la "Conferencia Mundial sobre la Educación Superior. La educación superior en el siglo XXI. Visión y acción”. París, Francia: UNESCO.

UNESCO-CRESALC (1997). La educación superior en el siglo XXI. Visión de América Latina y el Caribe. Caracas: CRESALC-UNESCO, Tomo-I. 